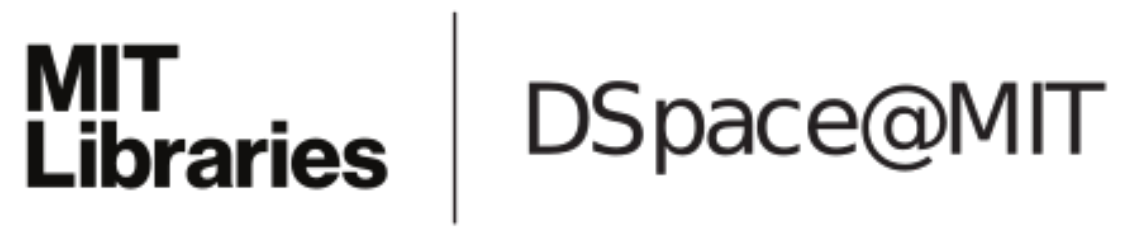

\author{
MIT Open Access Articles
}

Inter-nanomagnet force actuated deformable diffractive optics

The MIT Faculty has made this article openly available. Please share how this access benefits you. Your story matters.

Citation: Deterre, M. et al. "Inter-nanomagnet force actuated deformable diffractive optics." Optical MEMS and Nanophotonics, 2009 IEEE/LEOS International Conference on. 2009. 7-8. ( 2009 IEEE

As Published: http://dx.doi.org/10.1109/OMEMS.2009.5338631

Publisher: Institute of Electrical and Electronics Engineers

Persistent URL: http://hdl.handle.net/1721.1/54698

Version: Final published version: final published article, as it appeared in a journal, conference proceedings, or other formally published context

Terms of Use: Article is made available in accordance with the publisher's policy and may be subject to US copyright law. Please refer to the publisher's site for terms of use. 


\title{
Inter-nanomagnet force actuated deformable diffractive optics
}

\author{
M. Deterre, ${ }^{1}$ A. Nichol, ${ }^{1}$ Se Baek Oh, ${ }^{1}$ and G. Barbastathis ${ }^{1,2}$ \\ ${ }^{1}$ Massachusetts Institute of Technology \\ Department of Mechanical Engineering \\ ${ }^{2}$ Singapore-MIT Alliance for Research and Technology \\ (SMART) Centre \\ 77 Massachusetts Ave., Cambridge, MA 02139 \\ 3 Science Drive 2, Singapore 117543
}

\section{ABSTRACT}

We actively modulate the diffraction angle and wavefront of an optical beam by internanomagnet forces resulting from an external magnetic field applied to a free standing grating coated with nickel. Experimental results and simulations are presented.

\section{SUMMARY}

Tunable MEMS diffractive elements are interesting for a broad variety of applications, including displays, scanners, spectrometers, external cavity lasers, switches or wavelength tuning elements. Different techniques of tunable gratings have been implemented, such as piezoelectric ${ }^{1}$ or electrostatic ${ }^{2,3}$ actuation. In this work we demonstrate a freestanding grating coated with magnetic material, whose diffraction pattern is modified upon application of an external magnetic field. Previous work showed the folding, actuation and alignment of MEMS components including optical features using nanomagnets. ${ }^{4}$ Here we show the use of nanomagnets as a deformation agent for the element itself.

In the experiment, the grating (Fig. 2) was $200 \mu \mathrm{m}$ long, had a $800 \mathrm{~nm}$ pitch and was made of freestanding silicon nitride $\left(\mathrm{SiN}_{\mathrm{x}}, 400 \mathrm{~nm}\right)$ coated with nickel $(20 \mathrm{~nm})$. The fabrication process is shown in Fig. 1. On a $\mathrm{SiN}_{\mathrm{x}}$ membrane, grating lines were patterned with electron beam lithography at $30 \mathrm{KeV}$. After development of the electron beam resist, the nickel layer was wet etched using TFB type etchant. The resulting nickel acted then as an etch mask for the reactive ion etch (RIE), releasing a freestanding grating of silicon nitride and nickel.

When we apply a sufficiently strong in-plane magnetic field in a direction perpendicular to the grating, the grating lines in nickel are magnetized in the same direction as the field (Fig. 2 ). The grating lines are then attracted to each other and bend until the equilibrium between the elastic resistance and the magnetic force is reached. In a regular grating covering the entire sample, the global equilibrium position would not yield any bending as each line would be equally attracted by its two neighbors. Hence, several lines are removed on each side to break this equilibrium point and achieve the bowed state depicted in Fig. 2 schematically and in Fig. 3 in simulation.

In experiment, the grating was illuminated by an expanded doubled Nd:YAG laser beam and the intensity of the $1^{\text {st }}$ diffraction order was measured at the Fourier plane of a collector lens. We deformed the grating by moving a permanent magnet ( $0.5 \mathrm{~T}$ at its surface) manually near the grating. Without the external magnetic field, we observed the typical diffraction pattern resulting from a square grating. When the permanent magnet was brought to the proximity of the grating, the diffraction pattern changed, indicating a corresponding change in grating pitch, as shown in Fig. 4. (More images and videos are available at http://web.mit.edu/deterre/Public/omems09/) Due to physical constraints, the grooves of the grating not only translate but also bend as shown in the numerical simulation of Fig. 3; hence, the diffraction pattern shifts and also smears.

[1] Wong C W, Jeon Y, Barbastathis G and Kim S G, 2004 J. Microelectromech. Syst. 13998

[2] Shih W-C, Kim S-G and Barbastathis G, 2006 J. Microelectromech. Syst.15 763-9

[3] Tormen M, Peter Y-A, Niedermann P, Hoogerwerf A and Stanley R, 2006 J. Opt. A: Pure Appl. Opt. 8 S337-S340

[4] Nichol A, Arora W, Barbastathis G, 2006 J. Vac. Sci. Technol. B 24(6) 
After several cycles of actuation we observed a reversal in the displacement direction of the diffracted order. During the first few cycles the diffracted order moved away from the DC term indicating shortening of the grating pitch, as expected (Fig. 4(a)). However, in subsequent cycles we observed the opposite motion, indicating increase in the grating pitch (Fig. 4(b)). We interpret this phenomenon as due to residual magnetization or partial oxidation of the nickel magnets; a complete explanation of the cause of the reversal is still under investigation.

We are considering several additional improvements; for example, we can increase the magnetic force by coating thicker nickel films, or use grating patterns with flexures allowing parallel motion of the grating lines. This proof of concept shows that nanomagnets can actuate diffractive elements. This is a promising step toward the goal of an integrated tunable optical microsystem actuated by nanomagnets.

i)

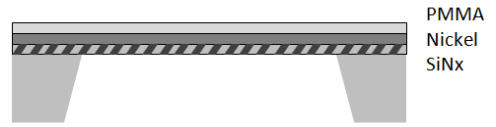

ii)

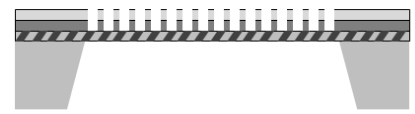

iii)

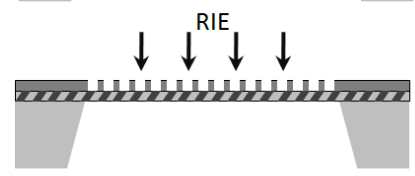

iv)

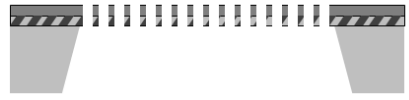

v)

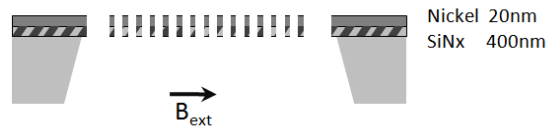

Figure 1:side view - i) Ni is evaporated and PMMA spun on a SiNx membrane. ii) The grating is written by electron-beam lithography. Nickel is wet etched after the development. iii),iv) The grating is released by a reactive ion etch of the SiNx. v) The grating is compressed when external magnetic field is applied.

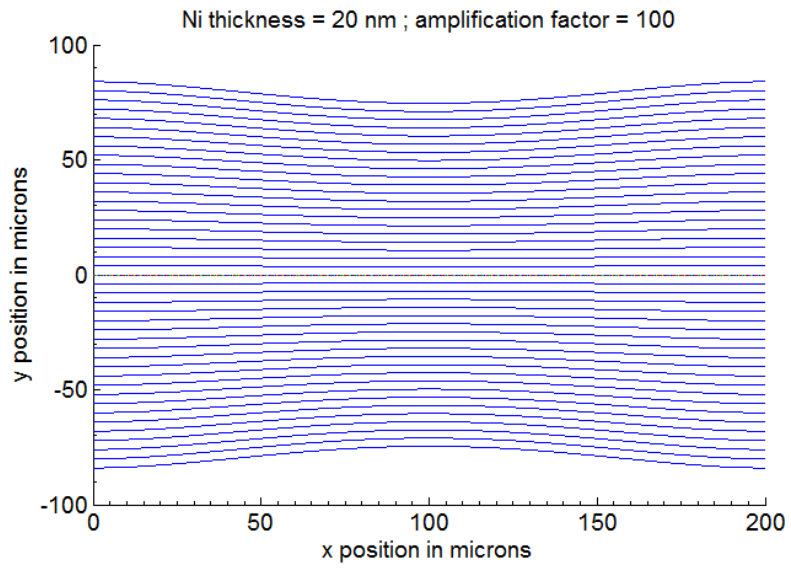

Figure 3: Simulation of the grating bending under magnetic actuation. For visualization purposes, every fifth line is drawn and the bending is amplified by a factor of 100 . i)

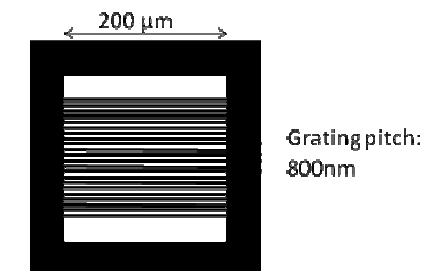

ii)

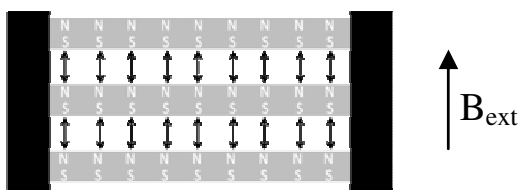

iii)

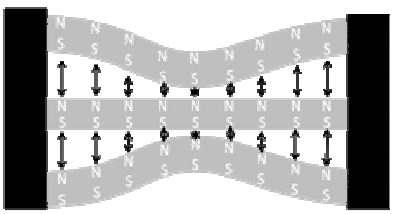

Figure 2: top view - i) Lines are removed from the grating edges to avoid the no-distortion equilibrium ii), iii) The grating lines are magnetized in plane perpendicularly to the grating. iii) Lines bow through magnetic interaction (amplified schematic).

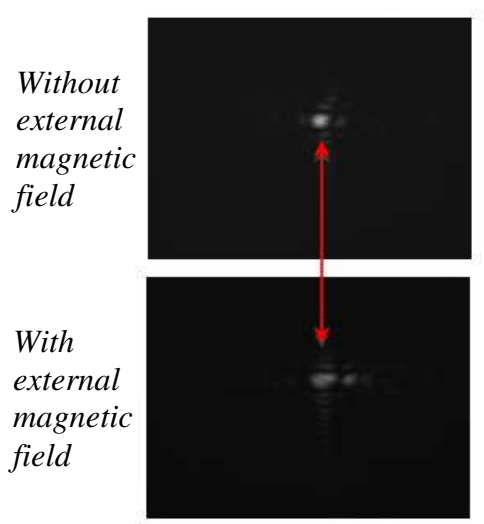

a)
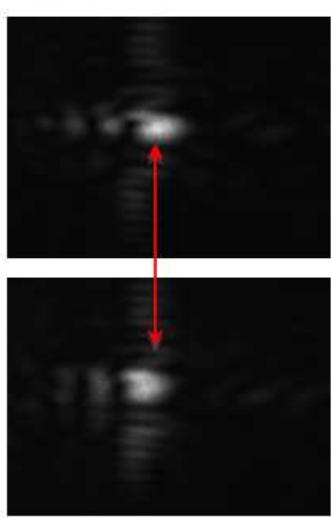

b)
Figure 4: Diffraction pattern for the first actuation cycles (a) and after direction reversal (b). The lateral shift of the diffraction pattern is approximately $200 m$ (40 pixels) for case (a) and -250 $m$ (50 pixels) for case (b), corresponding to $0.7 \mathrm{mrad}$ and -1.3 mrad respectively, whereas the simulation predicted 1 mrad. 